\title{
Simulação para a equação de transporte em domínio não homogêneo utilizando o método de Nyström
}

\author{
Luana Lazzari ${ }^{1}$ \\ Esequia Sauter ${ }^{2}$ \\ Fábio Souto de Azevedo ${ }^{3}$ \\ PPGMAp/UFRGS, Porto Alegre, RS
}

\begin{abstract}
Resumo. Neste trabalho resolvemos numericamente a equação de transporte em domínio não homogêneo com condições de contorno semi-refletivas através de duas metodologias. Na metodologia 1, determinamos a solução para a formulação integral do problema de trasporte em domínio não homogêneo enquanto, na metodologia 2, resolvemos um problema de transporte em meio homogêneo obtido do problema não homogêneo original através de uma mudança de variável. Ambas metodologias utilizam técnicas analíticas e computacionais para remover as singularidades dos operadores integrais e aplicam o método de Nyström para discretizar esses operadores. Nossos resultados numéricos são comparados com dados da literatura e também entre as próprias metodologias.
\end{abstract}

Palavras-chave. Equação Integral do Transporte, Operadores Singulares, Método de Nyström

\section{Introdução}

Aplicada em diferentes áreas, como física de reatores [6], medicina nuclear [5] e transferência radiativa [8], a equação de transporte é um modelo matemático que descreve a distribuição das partículas em um meio. Devido a importância dessa equação, diversas metodologias, tanto analíticas como computacionais, já foram desenvolvidas para solucionar esse problema. Uma forma equivalente da equação integro-diferencial de transporte é a chamada formulação integral. Esta formulação é determinada integrando a equação de transporte ao longo das linhas características e reescrevendo este problema como uma equação de Fredholm do segundo tipo. Este procedimento nos permite tratar com exatidão a variável angular, visto que a solução da equação integral, que é o fluxo escalar, depende somente das variáveis espaciais.

Entre as diversas metodologias de solução da equação integral de transporte, podemos citar o método de Nyström, o qual consiste basicamente em aproximar os operadores integrais por uma quadratura numérica e assim obter um sistema de equações algébricas. Os métodos integrais, como o método de Nyström, são conhecidos por produzirem resultados precisos para a equação de transporte em várias geometrias além de funcionarem muito bem quando o problema de transporte deve ser resolvida milhares de vezes com o mesmo conjunto de parâmetros e fontes diferentes [2]. Embora o método de Nyström tenha um custo computacional semelhante aos métodos em ordenadas discretas, existem situações em que ele pode realmente ter uma vantagem significativa no tempo de simulação sem perder a precisão.

\footnotetext{
${ }^{1}$ luana-lazzari@hotmail.com

2 esequia.sauter@ufrgs.br

3 fabio.azevedo@ufrgs.br
} 
O método de Nyström tem sido empregado com sucesso na solução numérica da equação do transporte em domínio homogêneo para diferentes geometrias [1,3,4]. Com base nisso, nós propomos neste trabalho estender a aplicação deste método integral para a equação de transporte em domínio não homogêneo.

A solução da equação de transporte é determinada aqui por meio de duas metodologias. A ideia base da metodologia 1 é discretizar a formulação integral do problema em domínio não homogêneo através do método de Nyström e da metodologia 2 é aplicar este método na formulação integral de um problema de trasporte em meio homogêneo que é determinado a partir de uma mudança de variável aplicada ao problema original. Em ambas metodologias são aplicadas técnicas analíticas e computacionais para tratar das singularidades dos operadores integrais.

A fim de validar as metodologias 1 e 2 comparamos nossos resultados numéricos com dados da literatura e entre as próprias metodologias. Simulamos o problema de transporte para os domínios homogêneo, como o problema apresentado no trabalho de Dalmolim et al. [4], multirregião, como proposto no trabalho de Nunes e Barros [9] e domínio não homogêneo, dado no trabalho de Garcia e Siewert [7]. Nas simulações geramos os resultados numéricos considerando as quadraturas de Gauss-Legendre e regra de Boole.

O problema de transporte unidimensional para domínio não homogêneo e condições de contorno semi-refletivas, solucionado nesse trabalho, é dado por

$$
\left\{\begin{array}{l}
\mu \frac{\partial}{\partial x} \Psi(x, \mu)+\sigma_{t}(x) \Psi(x, \mu)=\frac{\sigma_{s}(x)}{2} \int_{-1}^{1} \Psi\left(x, \mu^{\prime}\right) d \mu^{\prime}+S(x) \\
\Psi(0, \mu)=\rho_{0}(\mu) \Psi(0,-\mu)+\left(1-\rho_{0}(\mu)\right) B_{0}(\mu), \quad \mu>0 \\
\Psi(L, \mu)=\rho_{L}(\mu) \Psi(L,-\mu)+\left(1-\rho_{L}(\mu)\right) B_{L}(\mu), \quad \mu<0
\end{array}\right.
$$

onde $\Psi(x, \mu)$ é o fluxo angular de partículas, $0 \leq x \leq L$ é a variável espacial e $\mu$ é o cosseno do ângulo entre a direção de propagação das partículas e o eixo $x$. A função $S(x)$ é a fonte e $\sigma_{t}(x)$ e $\sigma_{s}(x)$ representam as seções de choque macroscópica total e de espalhamento, respectivamente. Estamos considerando as seguintes condições para as seções de choque $\sigma_{t}(x)>\sigma_{s}(x), \sigma_{t}(x)>0$ e $\sigma_{s}(x)>0$. Nas condições de contorno temos que $B_{0}$ e $B_{L}$ são as funções que representam a contribuição da fronteira, $0 \leq \rho_{0} \leq 1$ e $0 \leq \rho_{L} \leq 1$ são os coeficientes de reflexão.

\section{Metodologia 1}

Apresentamos aqui uma breve descrição dos procedimentos matemáticos que envolvem a metodologia 1, sendo estes a determinação da formulação integral do problema de transporte, remoção da singularidade do operador integral e discretização deste operador aplicando método de Nyström.

Iniciamos determinando a formulação integral do problema de transporte, dado em (1), a partir da definição do fluxo escalar de partículas dado por

$$
\Phi(x)=\frac{1}{2} \int_{-1}^{1} \Psi(x, \mu) d \mu=\frac{1}{2} \int_{0}^{1}[\Psi(x,-\mu)+\Psi(x, \mu)] d \mu .
$$

As funções $\Psi(x,-\mu)$ e $\Psi(x, \mu)$ são determinadas solucionando a equação (1), reescrita como

$$
\frac{\partial}{\partial x} \Psi(x, \mu)+\frac{\sigma_{t}(x)}{\mu} \Psi(x, \mu)=\frac{Q(x)}{\mu}
$$

onde $Q(x)=\sigma_{s}(x) \Phi(x)+S(x)$, pelo método do fator integrante juntamente com a aplicação das condições de contorno.

Determinados os fluxos angulares para $\mu>0$ e $\mu<0$ podemos escrever a equação para o fluxo escalar como $\Phi(x)=\left(L_{g} Q\right)(x)+\left(L_{b} B\right)(x)$ onde $L_{g} Q(x)=\int_{0}^{L} k(x, \tau) Q(\tau) d \tau$, cujo núcleo $k(x, \tau)$ é 
escrito da forma:

$$
\begin{gathered}
k(x, \tau)=\int_{0}^{1}\left[\frac{\rho_{L}(-\mu) e^{-\frac{1}{\mu}\left(\int_{x}^{L} \sigma_{t}(k) d k+\int_{\tau}^{L} \sigma_{t}(k) d k\right)}+\rho_{0}(\mu) e^{-\frac{1}{\mu}\left(\int_{0}^{\tau} \sigma_{t}(k) d k+\int_{0}^{x} \sigma_{t}(k) d k\right)}}{2 \mu\left(1-\rho_{0}(\mu) \rho_{L}(-\mu) e^{-\frac{2}{\mu} \int_{0}^{L} \sigma_{t}(k) d k}\right)}\right. \\
\left.+\frac{e^{-\frac{1}{\mu}\left|\int_{x}^{\tau} \sigma_{t}(k) d k\right|}}{2 \mu}+\frac{\rho_{0}(\mu) \rho_{L}(-\mu) e^{-\frac{2}{\mu} \int_{0}^{L} \sigma_{t}(k) d k}\left(e^{-\frac{1}{\mu} \int_{x}^{\tau} \sigma_{t}(k) d k}+e^{-\frac{1}{\mu} \int_{\tau}^{x} \sigma_{t}(k) d k}\right)}{2 \mu\left(1-\rho_{0}(\mu) \rho_{L}(-\mu) e^{-\frac{2}{\mu} \int_{0}^{L} \sigma_{t}(k) d k}\right)}\right] d \mu .
\end{gathered}
$$

e a função $L_{b} B(x)$ é dada por

$$
\begin{aligned}
L_{b} B(x) & =\int_{0}^{1} \frac{\left.e^{-\frac{1}{\mu} \int_{0}^{x} \sigma_{t}(k) d k}\left(\left(1-\rho_{0}(\mu)\right) B_{0}(\mu)\right)+e^{-\frac{1}{\mu} \int_{0}^{L} \sigma_{t}(k) d k}\left(1-\rho_{L}(-\mu)\right) B_{L}(-\mu) \rho_{0}(\mu)\right)}{2\left(1-\rho_{0}(\mu) \rho_{L}(-\mu) e^{-\frac{2}{\mu} \int_{0}^{L} \sigma_{t}(k) d k}\right)} \\
& +\frac{e^{-\frac{1}{\mu} \int_{x}^{L} \sigma_{t}(k) d k}\left(\left(1-\rho_{L}(-\mu)\right) B_{L}(-\mu)+e^{-\frac{1}{\mu} \int_{0}^{L} \sigma_{t}(k) d k}\left(1-\rho_{0}(\mu)\right) B_{0}(\mu) \rho_{L}(-\mu)\right)}{2\left(1-\rho_{0}(\mu) \rho_{L}(-\mu) e^{-\frac{2}{\mu} \int_{0}^{L} \sigma_{t}(k) d k}\right)} d \mu .
\end{aligned}
$$

Substituindo a definição da função $Q(x)$ na equação para o fluxo escalar obtemos,

$$
\Phi(x)=\int_{0}^{L} \sigma_{s}(\tau) k(x, \tau) \Phi(\tau) d \tau+g(x),
$$

onde $g(x)=L_{g} S(x)+L_{b} B(x)$. A função $k(x, \mu)$, núcleo do operador desta função, possui uma singularidade na diagonal, $\tau=x$. Tratamos esta singularidade aplicando a técnica de subtração de singularidades e com isso $\Phi(x)$ assume a seguinte forma:

$$
\Phi(x)=\int_{0}^{L} k(x, \tau)\left[\Phi(\tau) \sigma_{s}(\tau)-\Phi(x) \sigma_{s}(x)\right] d \tau+\Phi(x) \sigma_{s}(x) R(x)+g(x),
$$

onde $R(x)=\int_{0}^{L} k(x, \tau) d \tau$. Removida a singularidade aplicamos o método de Nyström para aproximar o operador integral da equação (7) e avaliamos a mesma em cada ponto $i=1, \ldots, N$ da malha

$$
\Phi\left(x_{i}\right) \approx \sum_{\substack{j=1 \\ j \neq i}}^{N} w\left(\tau_{j}\right) k\left(x_{i}, \tau_{j}\right)\left[\Phi\left(\tau_{j}\right) \sigma_{s}\left(\tau_{j}\right)-\Phi\left(x_{i}\right) \sigma_{s}\left(x_{i}\right)\right]+\Phi\left(x_{i}\right) \sigma_{s}(x) R\left(x_{i}\right)+g\left(x_{i}\right),
$$

onde $\left\{w\left(\tau_{j}\right)\right\}_{j=1}^{N}$ são os pesos da quadratura.

\section{Metodologia 2}

Na metodologia 2 iniciamos transformando o problema de transporte em domínio não homogêneo em um problema em meio homogêneo, através de uma mudança de variável. Na sequência determinamos a formulação integral desse novo problema, removemos, por meio de técnicas analíticas e computacionais, as singularidades do operador integral e discretizamos esse operador aplicando o método de Nyström.

A mudança de variável proposta nessa metodologia é dada pela função $y(x)=\int_{0}^{x} \sigma_{t}(\tau) d \tau$. Por $\sigma_{t}(x)$ ser uma função estritamente positiva, $y(x)$ é uma função crescente e portanto bijetora. Logo podemos aplicar mudança de variável no problema (1) e obter um problema em meio homogêneo

$$
\mu \frac{\partial}{\partial y} \bar{\Psi}(y, \mu)+\bar{\Psi}(y, \mu)=\frac{\bar{\sigma}_{s}(y)}{2 \bar{\sigma}_{t}(y)} \int_{-1}^{1} \bar{\Psi}\left(y, \mu^{\prime}\right) d \mu^{\prime}+\frac{\bar{S}(y)}{\bar{\sigma}_{t}(y)} .
$$


Seguindo os mesmo passos da metodologia 1 determinarmos a formulação integral da equação (9), a qual é dada da seguinte forma

$$
\bar{\Phi}(x)=\int_{0}^{\bar{L}} \bar{k}(y, \tau)\left(\frac{\bar{\sigma}_{s}(\tau)}{\bar{\sigma}_{t}(\tau)} \bar{\Phi}(\tau)-\frac{\bar{\sigma}_{s}(y)}{\bar{\sigma}_{t}(y)} \bar{\Phi}(y)\right) d \tau+\frac{\bar{\sigma}_{s}(y)}{\bar{\sigma}_{t}(y)} \bar{\Phi}(y) \bar{R}(y)+\bar{g}(y),
$$

onde $\bar{R}(y)=\int_{0}^{\bar{L}} \bar{k}(y, \tau) d \tau$ e $\bar{g}(y)=L_{g}\left(\frac{\bar{S}(y)}{\bar{\sigma}_{t}(y)}\right)+L_{b} \bar{B}(y)$

A função $L_{g} \bar{Q}(y)$ é definida por $L_{g} \bar{Q}(y)=\int_{0}^{\bar{L}} \bar{k}(y, \tau) \bar{Q}(\tau) d \tau$ e seu núcleo $\bar{k}(y, \tau)$ dado por

$$
\begin{gathered}
\bar{k}(y, \tau)=\frac{1}{2} \int_{0}^{1}\left[\frac{\rho_{\bar{L}}(-\mu) e^{-\frac{1}{\mu}(2 \bar{L}-\tau-y)}+\rho_{0}(\mu) \rho_{\bar{L}}(-\mu) e^{-\frac{1}{\mu}(2 \bar{L}+\tau-y)}}{\mu\left(1-\rho_{0}(\mu) \rho_{\bar{L}}(-\mu) e^{-\frac{2 \bar{L}}{\mu}}\right)}\right. \\
\left.\frac{+\rho_{0}(\mu) e^{-\frac{1}{\mu}(y+\tau)}+\rho_{0}(\mu) \rho_{\bar{L}}(-\mu) e^{-\frac{1}{\mu}(2 \bar{L}-\tau+y)}}{\mu\left(1-\rho_{0}(\mu) \rho_{\bar{L}}(-\mu) e^{-\frac{2 \bar{L}}{\mu}}\right)}+\frac{e^{-\frac{1}{\mu}|\tau-y|}}{\mu}\right] d \mu .
\end{gathered}
$$

A função $L_{b} \bar{B}(y)$ é definida como,

$$
\begin{aligned}
L_{b} \bar{B}(y)=\int_{0}^{1}\left[\frac{\left(1-\rho_{\bar{L}}(-\mu)\right) B_{\bar{L}}(-\mu) e^{-\frac{1}{\mu}(\bar{L}-y)}+\left(1-\rho_{0}(\mu)\right) B_{0}(\mu) \rho_{\bar{L}}(-\mu) e^{-\frac{1}{\mu}(2 \bar{L}-y)}}{2\left(1-\rho_{0}(\mu) \rho_{\bar{L}}(-\mu) e^{-\frac{2 \bar{L}}{\mu}}\right)}\right. \\
\left.+\frac{\left(1-\rho_{0}(\mu) B_{0}(\mu)\right) e^{-\frac{y}{\mu}}+\left(1-\rho_{\bar{L}}(-\mu)\right) B_{\bar{L}}(-\mu) \rho_{0}(\mu) e^{-\frac{1}{\mu}(y+\bar{L})}}{2\left(1-\rho_{0}(\mu) \rho_{\bar{L}}(-\mu) e^{-\frac{2 \bar{L}}{\mu}}\right)}\right] d \mu .
\end{aligned}
$$

Aproximando o operador integral da equação (10) pelo método de Nyström e avaliando esta equação em cada ponto $i$ da malha,com $i=1, \ldots, N$, obtemos a seguinte equação para o fluxo escalar

$$
\bar{\Phi}\left(y_{i}\right) \approx \sum_{\substack{j=1 \\ j \neq i}}^{N} w\left(\tau_{j}\right) \bar{k}\left(y_{i}, \tau_{j}\right)\left[\bar{\Phi}\left(\tau_{j}\right) \frac{\bar{\sigma}_{s}\left(\tau_{j}\right)}{\bar{\sigma}_{t}\left(\tau_{j}\right)}-\bar{\Phi}\left(y_{i}\right) \frac{\bar{\sigma}_{s}\left(y_{i}\right)}{\bar{\sigma}_{t}\left(y_{i}\right)}\right]+\bar{\Phi}\left(y_{i}\right) \frac{\bar{\sigma}_{s}\left(y_{i}\right)}{\bar{\sigma}_{t}\left(y_{i}\right)} \bar{R}\left(y_{i}\right)+\bar{g}\left(y_{i}\right) .
$$

\section{Resultados numéricos}

Com o objetivo de validar nossos algoritmos, nesta seção simulamos os problemas em domínios homogêneo, multirregião e não homogêneo usando as metodologias 1 e 2 e comparamos nossos resultados com aqueles encontrados na literatura. As quadraturas numéricas utilizadas nas simulações foram Gauss-Legendre e regra de Boole.

Os resultados numéricos para o problema em meio homogêneo são comparados com o trabalho desenvolvido por Dalmolin et al. [4], no qual a equação de trasporte é solucionada pelo método de Nyström. Para o caso multirregião com três camadas simulamos um problema do trabalho de Nunes e Barros [9] no qual o sistema de equações $S_{8}$ foi solucionado método Diamond Difference (DD). Por fim, apresentamos uma simulação para o domínio não homogêneo, problema proposto por Garcia e Siewert [7], cuja solução é determinada pelo método $F_{N}$.

Os parâmetros para cada problema simulado são:

- Problema em domínio homogêneo: $L=1 \mathrm{~cm}, \sigma_{t}(x)=\sigma_{s}(x)=1 \mathrm{~cm}^{-1}, \rho_{0}(\mu)=\rho_{L}(\mu)=0$, $B_{0}(\mu)=B_{L}(\mu)=0.125$ e $S(x)=0.125$.

- Problema multirregião: $L=40 \mathrm{~cm}, \Psi(0, \mu)=1, \Psi(L, \mu)=0$ e $\sigma_{t}(x)=1$. As funções $\sigma_{s}(x)$ e $S(x)$ para cada região são apresentadas na tabela 1. 
- Problema em domínio não homogêneo: $L=5 \mathrm{~cm}, \sigma_{t}(x)=1 \mathrm{~cm}^{-1}, \sigma_{s}(x)=\exp (-x / s) \mathrm{cm}^{-1}$, $\rho_{0}(\mu)=\rho_{L}(\mu)=B_{L}(\mu)=0, B_{0}(\mu)=1$ e $S(x)=0$.

Tabela 1: Parâmetros do problema multirregião.

\begin{tabular}{cccc}
\hline Região & 1 & 2 & 3 \\
\hline $\mathrm{S}(\mathrm{x})$ & 0 & 0 & 1 \\
$\sigma_{s}(x)$ & 0.97 & 0.95 & 0.99 \\
Espessura & 15 & 15 & 10 \\
\hline
\end{tabular}

Tabela 2: Resultados numéricos para o problema em domínio homogêneo.

\begin{tabular}{cccccccc}
\hline x & Nyström & $N=401^{\mathrm{a}}$ & $N=801^{\mathrm{a}}$ & $N=1601^{\mathrm{a}}$ & $N=401^{\mathrm{b}}$ & $N=801^{\mathrm{b}}$ & $N=1601^{\mathrm{b}}$ \\
\hline 0.0 & 0.516842 & 0.516842 & 0.516842 & 0.516842 & 0.516841 & 0.516841 & 0.516842 \\
0.1 & 0.600637 & 0.600637 & 0.600637 & 0.600637 & 0.600637 & 0.600637 & 0.600637 \\
0.2 & 0.647999 & 0.647999 & 0.647999 & 0.647999 & 0.647999 & 0.647999 & 0.647999 \\
0.3 & 0.678718 & 0.678718 & 0.678718 & 0.678718 & 0.678718 & 0.678718 & 0.678718 \\
0.4 & 0.696308 & 0.696308 & 0.696308 & 0.696308 & 0.696308 & 0.696308 & 0.696308 \\
0.5 & 0.702056 & 0.702056 & 0.702056 & 0.702056 & 0.702055 & 0.702055 & 0.702056 \\
\hline
\end{tabular}

a. Quadratura de Gauss-Legendre.

b. Regra de Boole.

Tabela 3: Resultados numéricos para o problema multirregião aplicando quadratura de Gauss-Legendre.

\begin{tabular}{cccccccc}
\hline$x$ & $D D$ & $N=501$ & $N=1001$ & $N=2001$ & $N=4001$ & $N=8001$ & $N=16001$ \\
\hline 0.0 & 0.852638 & 0.852638 & 0.852639 & 0.852638 & 0.852638 & 0.852638 & 0.852638 \\
20.0 & 0.481766 & 0.482850 & 0.484256 & 0.482214 & 0.482542 & 0.482026 & 0.482108 \\
40.0 & 7.090416 & 7.093101 & 7.095029 & 7.091948 & 7.092451 & 7.091686 & 7.091813 \\
\hline
\end{tabular}

Tabela 4: Resultados numéricos para o problema multirregião aplicando regra de Boole.

\begin{tabular}{cccccccc}
\hline$x$ & $D D$ & $N=501$ & $N=1001$ & $N=2001$ & $N=4001$ & $N=8001$ & $N=16001$ \\
\hline 0.0 & 0.852638 & 0.852831 & 0.852693 & 0.852653 & 0.852642 & 0.852639 & 0.852638 \\
20.0 & 0.481766 & 0.476498 & 0.480935 & 0.481354 & 0.481662 & 0.481815 & 0.481892 \\
40.0 & 7.090416 & 7.073885 & 7.087380 & 7.089942 & 7.090936 & 7.091313 & 7.091469 \\
\hline
\end{tabular}

Na tabela 2 apresentamos os resultados numéricos para o problema de transporte em domínio homogêneo para as quadraturas de Gauss-Legendre e regra de Boole. Para ambas quadraturas obtivemos seis dígitos de precisão quando comparada com a solução apresentada em [4]. Com relação ao desempenho dos esquemas quadraturas observamos que para a quadradura de GaussLegendre a solução convergiu em $\mathrm{N}=401$ pontos enquanto que na regra de Boole em $\mathrm{N}=1601$.

Para o caso multirregião consideramos um problema com três camadas, apresentado no trabalho de Nunes e Barros [9], e simulamos aplicando as quadraturas de Gauss-Legendre e regra de Boole, tabelas 3 e 4 respectivamente. Os resultados obtidos por ambas quadraturas tem a mesma ordem 
Tabela 5: Fluxo angular para $x=0, \Psi(0,-\mu)$.

\begin{tabular}{ccccccccc}
\hline$\mu$ & $s=1^{\mathrm{a}}$ & $s=1^{\mathrm{b}}$ & $s=10^{\mathrm{a}}$ & $s=10^{\mathrm{b}}$ & $s=100^{\mathrm{a}}$ & $s=100^{\mathrm{b}}$ & $s=1000^{\mathrm{a}}$ & $s=1000^{\mathrm{b}}$ \\
\hline 0.05 & 0.58966 & 0.58966 & 0.76081 & 0.76081 & 0.863400 & 0.863400 & 0.89361 & 0.89361 \\
0.1 & 0.53112 & 0.53112 & 0.73398 & 0.73398 & 0.85028 & 0.85028 & 0.88319 & 0.88319 \\
0.2 & 0.44328 & 0.44328 & 0.68632 & 0.68632 & 0.82523 & 0.82523 & 0.86410 & 0.86410 \\
0.3 & 0.38031 & 0.38031 & 0.64418 & 0.64418 & 0.80181 & 0.80181 & 0.84606 & 0.84606 \\
0.4 & 0.33296 & 0.33296 & 0.60647 & 0.60647 & 0.77946 & 0.77946 & 0.82857 & 0.82857 \\
0.5 & 0.29609 & 0.29609 & 0.57257 & 0.57257 & 0.75799 & 0.75799 & 0.81143 & 0.81143 \\
0.6 & 0.26656 & 0.26656 & 0.54197 & 0.54197 & 0.73730 & 0.73730 & 0.79455 & 0.79455 \\
0.7 & 0.24239 & 0.24239 & 0.51427 & 0.51427 & 0.71733 & 0.71733 & 0.77788 & 0.77788 \\
0.8 & 0.22223 & 0.22223 & 0.48910 & 0.48910 & 0.69805 & 0.69805 & 0.76139 & 0.76139 \\
0.9 & 0.20517 & 0.20517 & 0.46615 & 0.46615 & 0.67943 & 0.67943 & 0.74510 & 0.74510 \\
1.0 & 0.19055 & 0.19055 & 0.44517 & 0.44517 & 0.66146 & 0.66146 & 0.72904 & 0.72904 \\
\hline
\end{tabular}

a. Metodologias 1 e 2 .

b. Garcia e Siewert [7].

Tabela 6: Fluxo angular para $x=L, \Psi(L, \mu)$.

\begin{tabular}{ccccccc}
\hline$\mu$ & $s=1^{\mathrm{a}}$ & $s=1^{\mathrm{b}}$ & $s=10^{\mathrm{a}}$ & $s=10^{\mathrm{b}}$ & $s=100^{\mathrm{a}}$ & $s=100^{\mathrm{b}}$ \\
\hline 0.05 & $0.6075 \mathrm{E}-5$ & $0.6075 \mathrm{E}-5$ & $0.58031 \mathrm{E}-2$ & $0.58031 \mathrm{E}-2$ & $0.62883 \mathrm{E}-1$ & $0.62883 \mathrm{E}-1$ \\
0.1 & $0.69252 \mathrm{E}-5$ & $0.69252 \mathrm{E}-5$ & $0.63702 \mathrm{E}-2$ & $0.63702 \mathrm{E}-2$ & $069024 \mathrm{E}-1$ & $069024 \mathrm{E}-1$ \\
0.2 & $0.96423 \mathrm{E}-5$ & $0.96423 \mathrm{E}-5$ & $0.76183 \mathrm{E}-2$ & $0.76183 \mathrm{E}-2$ & $0.80567 \mathrm{E}-1$ & $0.80567 \mathrm{E}-1$ \\
0.3 & $0.16234 \mathrm{E}-4$ & $0.16234 \mathrm{E}-4$ & $0.91482 \mathrm{E}-2$ & $0.91482 \mathrm{E}-2$ & $0.91918 \mathrm{E}-1$ & $0.91918 \mathrm{E}-1$ \\
0.4 & $0.43858 \mathrm{E}-4$ & $0.43858 \mathrm{E}-4$ & $0.11119 \mathrm{E}-1$ & $0.11119 \mathrm{E}-1$ & 0.14012 & 0.14012 \\
0.5 & $0.16937 \mathrm{E}-3$ & $0.16937 \mathrm{E}-3$ & $0.13725 \mathrm{E}-1$ & $0.13725 \mathrm{E}-1$ & 0.11523 & 0.11523 \\
0.6 & $0.57347 \mathrm{E}-3$ & $0.57347 \mathrm{E}-3$ & $0.17183 \mathrm{E}-1$ & $0.17183 \mathrm{E}-1$ & 0.12744 & 0.12744 \\
0.7 & $0.15128 \mathrm{E}-2$ & $0.15128 \mathrm{E}-2$ & $0.21680 \mathrm{E}-1$ & $0.21680 \mathrm{E}-1$ & 0.14010 & 0.14010 \\
0.8 & $0.32437 \mathrm{E}-2$ & $0.32437 \mathrm{E}-2$ & $0.27331 \mathrm{E}-1$ & $0.27331 \mathrm{E}-1$ & 0.15319 & 0.15319 \\
0.9 & $0.59604 \mathrm{E}-2$ & $0.59604 \mathrm{E}-2$ & $0.34166 \mathrm{E}-1$ & $0.34166 \mathrm{E}-1$ & 0.16667 & 0.16667 \\
1.0 & $0.97712 \mathrm{E}-2$ & $0.97712 \mathrm{E}-2$ & $0.42142 \mathrm{E}-1$ & $0.42142 \mathrm{E}-1$ & 0.18047 & 0.18047 \\
\hline
\end{tabular}

a. Metodologias 1 e 2 .

b. Garcia e Siewert [7].

de precisão aos determinados pelo método DD, método aplicado na solução do sistemas de equações $S_{N}$ com ordem de discretização angular $N=8$, a qual é considerada baixa para conseguir uma boa precisão na solução do problema. Novamente a solução para a quadratura de Gauss-Legendre convergiu mais rapidamente que a solução obtida pela regra de Boole. Os problemas em domínio homogênea e multirregião foram simulados utilizando as metodologias 1 e 2 as quais resultaram em soluções iguais no entanto, a metodologia 2 apresentou melhor desempenho computacional.

Para o problema de transporte em domínio não homogêneo, tabelas 5 e 6 , comparamos nossos resultados com os apresentados no trabalho de Garcia e Siewert [7]. Para todos os valores numéricos de s que consideramos nas simulações, obtemos que para ambas metodologia e quadraturas numéricas, Gauss-Legendre e regra de Boole, as soluções apresentaram a mesma ordem de precisão que os dados da literatura. 


\section{Conclusões}

Neste trabalho determinamos a solução numérica para a equação de transporte em domínio não homogêneo com condições de contorno semi-refletivas através de duas metologias. Nas simulações considerando o problema de transporte para os domínios homogêneo, multirregião e não homogêneo. Em todos os casos, as duas metodologias foram eficientes na determinação da solução dos problemas, porém, a metodologia 2, na qual aplicamos a mudança de variável, os custos computacionais foram menores. Com relação a desempenho das quadraturas numéricas aplicadas na solução do problema a quadratura de Gauss-Legendre apresentou melhor desempenho numérico. No entanto, quando consideramos um número de pontos suficientemente grande os resultados numéricos, para a maioria dos casos testados, tiveram a mesma ordem de precisão.

\section{Agradecimentos}

Luana Lazzari agradece o suporte pela bolsa de doutorado do CNPq (Brasil).

\section{Referências}

[1] Azevedo, F., Sauter, E., Konzen, P.H. A., Thompson, M., Barichello, L. B. Integral formulation and numerical simuations for the neutron transport equation in x-y geoemtry. Annals of Nuclear Energy. 112: 735-747, 2018. DOI:10.1016/j.anucene.2017.10.017.

[2] Azevedo, F., Sauter, E., Thompson, M., Vilhena, M. T. Existence theory and simulations for one-dimensional radiative flows. Annals of Nuclear Energy.38:1115-1124, 2011. DOI: 10.1016/j.anucene.2010.12.014.

[3] Bublitz, C., de Azevedo, F. S., Sauter, E. Nyström method applied to the transport equation in an axisymmetric cylinder. Annals of Nuclear Energy.148: 107701, 2020. DOI: 10.1016/j.anucene.2020.107701.

[4] Dalmolin, D., Azevedo, F. S., Sauter, E. Nyström method in transport equation, Proceedings of INAC 2017 International Nuclear Atlantic Conference, 2017. ISBN: 978-05-99141-07-6.

[5] Demarco, G. Simulação Monte Carlo em terapia de câncer por Captura de nêutrons pelo Boro (BNCT) utilizando a plataforma GEANT4. Dissertação de Mestrado, UFRGS, 2009.

[6] Duderstadt, J. J., Hamilton, L. J. Nuclear Reactor Analysis. John Wiley, New York, 1976.

[7] Garcia, R. D. M., Siewert, C. E. Radiative transfer in finite inhomogeneous plane-parallel atmospheres. Journal of Quantitative Spectroscopy e Radiative Transfer. Printed in Great Britain, 141-148, 1982. DOI:10.1006/0022-4073(82)90134-0.

[8] Machado, R. S. Uma solução para o problema de transferência radiativa pelo método $L T S_{N}$ com albedo variável. Dissertação de Mestrado, UFRGS, 2008.

[9] Nunes, C. E. A., Barros, R. C. Aplicativo computacional para cálculos de blindagem com modelo de transporte $s_{n}$ unidimensional e monoenergético, Proceedings of INAC 2009 International Nuclear Atlantic Conference, 2009. ISBN: 978-85-99141-03-8. 